\title{
ON CHAOTIC BEHAVIOR OF THE P-ADIC GENERALIZED ISING MAPPING AND ITS APPLICATION
}

\author{
FARRUKH MUKHAMEDOV, HASAN AKIN, AND MUTLAY DOGAN
}

\begin{abstract}
In the present paper, by conducting research on the dynamics of the $p$-adic generalized Ising mapping corresponding to renormalization group associated with the $p$-adic Ising-Vannemenus model on a Cayley tree, we have determined the existence of the fixed points of a given function. Simultaneously, the attractors of the dynamical system have been found. We have come to a conclusion that the considered mapping is topologically conjugate to the symbolic shift which implies its chaoticity and as an application, we have established the existence of periodic $p$-adic Gibbs measures for the $p$-adic Ising-Vannemenus model.
\end{abstract}

Mathematics Subject Classification: 46S10, 82B26, 12J12, 39A70, 47H10, 60K35.

Key words: $p$-adic numbers; $p$-adic dynamical system; chaos; periodic.

\section{INTRODUCTION}

In [9] the thermodynamic behavior of the central site of an Ising spin system with ferromagnetic nearest-neighbor interactions on a Cayley tree was studied by recursive methods which consequently opened new perspectives between the recursion approach and the theory of dynamical systems. The existence of the phase transition is closely connected to the existence of the chaotic behavior of the associated dynamical system which is governed by the Ising-Potts function. Investigating the dynamics of this function has been the object of no small amount of study in the real and complex settings. This deceptively simple family of rational functions has given rise to a surprising number of interesting dynamical features (see for example $[6,10,16,27]$ ). Therefore, the combination of statistical mechanics tools and methods adopted from dynamical systems are one of the most promising directions in the theory of phase transitions. One of such tools is the renormalization group (RG) which has had a profound impact on modern statistical physics. This method appeared after Wilsons seminal work in the early 1970's [45], based also on the ground breaking foundations laid by Kadanoff, Widom, Michael Fisher [15].

On the other hand, there are many investigations that have been conducted to discuss and debate the question due to the assumption that $p$-adic numbers provide a more exact and more adequate description of microworld phenomena [20,43]) Consequently, various models in physics described in the language of $p$-adic analysis (see for example, $[1,3])$ ), and numerous applications of such an analysis to mathematical physics have been studied in [20,21, 24]. These investigations proposed to study new probability models (namely $p$-adic probability), which cannot be described using ordinary Kolmogorov's probability theory [22]. Using that, $p$-adic measure theory in $[28,29,37]$ the theory of $p$-adic statistical mechanics has been been developed. For complete review of the $p$-adic mathematical physics we refer to [8].

On the other hand, recently, polynomials and rational maps of $p$-adic numbers $\mathbb{Q}_{p}$ have been studied as dynamical systems over this field $[4,5]$. It turns out that these $p$-adic dynamical systems are quite different to the dynamical systems in Euclidean spaces (see for example, [2, 12, 11, 23, 26, 39] and their bibliographies therein). In theoretical physics, the interest in $p$-adic dynamical systems was started with the development of $p$-adic models $[28,30,23]$. In these investigations, the importance

Date: October 14, 2018. 
of detecting chaos was stressed in the $p$-adic setting [19, 41, 46]. In [29] the renormalization group method has been developed to study phase transitions for several $p$-adic models on Cayley trees. In [30] we have studied some particular cases of the Ising-Potts function and showed its chaoticity.

So presently, we study dynamics of a $p$-adic rational mapping ${ }^{1}$ (see (3.2)) the generalized Ising mapping associated with the Ising-Vannimenus model on a Cayley tree [32]. The existence of the phase transition for this model has been investigated in $[32,34]$. In section 3 we study the existence of fixed points of the mentioned mapping. In section 4 the dynamical behavior of the fixed points is explored and particularly, the basin of attraction of the attractive fixed point is described. In section 5, we show that the dynamical system (3.2) is topologically conjugate to the full shift, and hence it is chaotic. The obtained result opens certain perspectives in the study of generalized self-similar sets in the $p$-adic setting [31]. As an application of the result of section 5 , in the last section 6 , we illustrate the existence of periodic $p$-adic Gibbs measures for the Ising-Vannimenus model. Note that a construction of 2-periodic p-adic Gibbs measures has been given in [34], but using that construction, it is extremely difficult to find other kinds of periodic measures. Note that, in the $p$-adic setting, due to the lack of convex structure of the set of $p$ - adic Gibbs measures, it is quite difficult to constitute a phase transition with some features of the set of $p$-adic Gibbs measures. The result of section 6 implies that the set of $p$-adic Gibbs measures is huge. Moreover, the advantage of the present work allows us to find lots of periodic Gibbs measures. We point out that some numerical simulations predict [42] the chaotic behavior of the mapping in the real setting, but these are no rigorous proofs of this kind of fact.

\section{Preliminaries}

2.1. $p$-adic numbers. In what follows $p$ will be a fixed prime number, and by $\mathbb{Q}_{p}$ it is denoted the field of $p$-adic numbers, which is a completion of the rational numbers $\mathbb{Q}$ with respect to the norm $|\cdot|_{p}: \mathbb{Q} \rightarrow \mathbb{R}$ given by

$$
|x|_{p}=\left\{\begin{array}{cc}
p^{-r} & x \neq 0 \\
0, & x=0
\end{array}\right.
$$

here, $x=p^{r} \frac{m}{n}$ with $r, m \in \mathbb{Z}, n \in \mathbb{N},(m, p)=(n, p)=1$. The number $r$ is called the $p$-order of $x$ and it is denoted by $\operatorname{ord}_{p}(x)=r$. The absolute value $|\cdot|_{p}$, is non-Archimedean, meaning that it satisfies the ultrametric triangle inequality $|x+y|_{p} \leq \max \left\{|x|_{p},|y|_{p}\right\}$.

Any $p$-adic number $x \in \mathbb{Q}_{p}, x \neq 0$ can be uniquely represented in the form

$$
x=p^{\text {ord }}(x)\left(x_{0}+x_{1} p+x_{2} p^{2}+\ldots\right),
$$

where $x_{j}$ are integers such that $0 \leq x_{j} \leq p-1$ for all $j=0,1,2, \ldots$ and $x_{0}>0$, In this case $|x|_{p}=p^{-\operatorname{ord}_{p}(x)}$.

Lemma 2.1. Let $p \geq 3, a \in \mathbb{Q}_{p}$ and $a=p^{\operatorname{ord}_{p}(a)}\left(a_{0}+a_{1} p+a_{2} p^{2}+\ldots\right)$. Then $\sqrt{a}$ exists in $\mathbb{Q}_{p}$ if and only if

i) $\operatorname{ord}_{p}(a) \in 2 \mathbb{Z}_{p}$

ii) $x^{2} \equiv a_{0}(\operatorname{modp})$ has a solution in $\mathbb{Z}$.

In what follows, for the sake of simplicity, we always assume that $p \geq 3$, since $p=2$ is considered as a pathological case.

For each $a \in \mathbb{Q}_{p}, r>0$ we denote

$$
B_{r}(a)=\left\{x \in \mathbb{Q}_{p}:|x-a|_{p}<r\right\}, \quad S_{r}(a)=\left\{x \in \mathbb{Q}_{p}:|x-a|_{p}=r\right\}
$$

\footnotetext{
${ }^{1}$ We note that the dynamical properties of the fixed points of some $p$-adic rational maps have been studied in $[4,5,14,17,35,38]$. Some results on the global structure of rational maps on $\mathbb{Q}_{p}$ can be found in $[7,44]$.
} 
and the set of all $p$-adic integers

$$
\mathbb{Z}_{p}=\left\{x \in \mathbb{Q}_{p}:|x|_{p} \leq 1\right\} .
$$

The set $\mathbb{Z}_{p}^{*}=\mathbb{Z}_{p} \backslash p \mathbb{Z}_{p}$ is called a set of $p$-adic units. Recall that the $p$-adic logarithm is defined by the series

$$
\log _{p}(x)=\log _{p}(1+(x-1))=\sum_{n=1}^{\infty}(-1)^{n+1} \frac{(x-1)^{n}}{n},
$$

which converges for every $x \in B_{1}(1)$. And the $p$-adic exponential is defined by

$$
\exp _{p}(x)=\sum_{n=0}^{\infty} \frac{x^{n}}{n !}
$$

which converges for every $x \in B_{p^{-1 /(p-1)}}(0)$. Note that, in the considered setting (i.e. $p \geq 3$ ), due to the discreteness of the norm, we have $B_{p^{-1 /(p-1)}}(0)=B_{1}(0)$.

Lemma 2.2. ([25],[43]) Let $x \in B_{1}(0)$ then we have

$$
\left|\exp _{p}(x)\right|_{p}=1, \quad\left|\exp _{p}(x)-1\right|_{p}=|x|_{p}<1, \quad\left|\log _{p}(1+x)\right|_{p}=|x|_{p}<p^{-1 /(p-1)}
$$

and

$$
\log _{p}\left(\exp _{p}(x)\right)=x, \quad \exp _{p}\left(\log _{p}(1+x)\right)=1+x
$$

Denote

$$
\mathcal{E}_{p}=\left\{x \in \mathbb{Q}_{p}:|x-1|_{p}<1\right\} .
$$

Using Lemma 2.2 one can prove the following facts.

Lemma 2.3. [34] The set $\mathcal{E}_{p}$ has the following properties:

(1) $\mathcal{E}_{p}$ is a group under multiplication;

(2) $|a-b|_{p}<1$ for all $a, b \in \mathcal{E}_{p}$;

(3) if $a, b \in \mathcal{E}_{p}$ then it holds $|a+b|_{p}=1$;

(4) if $a \in \mathcal{E}_{p}$, then there is an element $h \in B\left(0, p^{-1 /(p-1)}\right)$ such that $a=\exp _{p}(h)$;

(5) if $a \in \mathcal{E}_{p}$, then $\sqrt{a} \in \mathcal{E}_{p}$.

Note that the basics of $p$-adic analysis, $p$-adic mathematical physics are explained in [20, 25].

2.2. Dynamical systems in $\mathbb{Q}_{p}$. In this subsection we recall some standard terminology of the theory of dynamical systems (see for example [23]).

A function $f: B_{r}(a) \rightarrow \mathbb{Q}_{p}$ is said to be analytic if it can be represented by

$$
f(x)=\sum_{n=0}^{\infty} f_{n}(x-a)^{n}, \quad f_{n} \in \mathbb{Q}_{p},
$$

which converges uniformly on the ball $B_{r}(a)$.

Consider a dynamical system $(f, B)$ in $\mathbb{Q}_{p}$, where $f: B \rightarrow B$ is an analytic function and $B=B_{r}(a)$ or $\mathbb{Q}_{p}$. Denote $x^{(n)}=f^{n}\left(x^{(0)}\right)$, where $x^{(0)} \in B$ and $f^{n}(x)=\underbrace{f \circ \cdots \circ f(x)}_{n}$. If $f\left(x^{(0)}\right)=x^{(0)}$ then $x^{(0)}$ is called a fixed point. A fixed point $x^{(0)}$ is called an attractor if there exists a neighborhood $U\left(x^{(0)}\right)(\subset B)$ of $x^{(0)}$ such that for all points $y \in U\left(x^{(0)}\right)$ it holds that $\lim _{n \rightarrow \infty} y^{(n)}=x^{(0)}$, where $y^{(n)}=f^{n}(y)$. If $x^{(0)}$ is an attractor then its basin of attraction is

$$
A\left(x^{(0)}\right)=\left\{y \in \mathbb{Q}_{p}: y^{(n)} \rightarrow x^{(0)}, n \rightarrow \infty\right\} .
$$

A fixed point $x^{(0)}$ is called repeller if there exists a neighborhood $U\left(x^{(0)}\right)$ of $x^{(0)}$ such that $\mid f(x)-$ $\left.x^{(0)}\right|_{p}>\left|x-x^{(0)}\right|_{p}$ for $x \in U\left(x^{(0)}\right), x \neq x^{(0)}$. 
Let $x^{(0)}$ be a fixed point of an analytic function $f(x)$. Set

$$
\lambda=\frac{d}{d x} f\left(x^{(0)}\right)
$$

The point $x^{(0)}$ is called attracting if $0 \leq|\lambda|_{p}<1$, indifferent if $|\lambda|_{p}=1$, and repelling if $|\lambda|_{p}>1$.

2.3. $p$-adic sub-shift. Let $f: X \rightarrow \mathbb{Q}_{p}$ be a map from a compact open set $X$ of $\mathbb{Q}_{p}$ into $\mathbb{Q}_{p}$. We assume that (i) $f^{-1}(X) \subset X$; (ii) $X=\cup_{j \in I} B_{r}\left(a_{j}\right)$ can be written as a finite disjoint union of balls of centers $a_{j}$ and of the same radius $r$ such that for each $j \in I$ there is an integer $\tau_{j} \in \mathbb{Z}$ such that

$$
|f(x)-f(y)|_{p}=p^{-\tau_{j}}|x-y|_{p}, \quad x, y \in B_{r}\left(a_{j}\right) .
$$

For such a map $f$, define its Julia set by

$$
J_{f}=\bigcap_{n=0}^{\infty} f^{-n}(X) .
$$

It is clear that $f^{-1}\left(J_{f}\right)=J_{f}$ and then $f\left(J_{f}\right) \subset J_{f}$.

Following [12] the triple $\left(X, J_{f}, f\right)$ is called a $p$-adic weak repeller if all $\tau_{j}$ in (2.5) are nonnegative, but at least one is positive. We call it a $p$-adic repeller if all $\tau_{j}$ in $(2.5)$ are positive. For any $i \in I$, we let

$$
I_{i}:=\left\{j \in I: B_{r}\left(a_{j}\right) \cap f\left(B_{r}\left(a_{i}\right)\right) \neq \varnothing\right\}=\left\{j \in I: B_{r}\left(a_{j}\right) \subset f\left(B_{r}\left(a_{i}\right)\right)\right\}
$$

(the second equality holds because of the expansiveness and the ultrametric property). Then define a matrix $A=\left(a_{i j}\right)_{I \times I}$, called incidence matrix as follows

$$
a_{i j}= \begin{cases}1, & \text { if } j \in I_{i} \\ 0, & \text { if } j \notin I_{i}\end{cases}
$$

If $A$ is irreducible, we say that $\left(X, J_{f}, f\right)$ is transitive. Here the irreducibility of $A$ means, for any pair $(i, j) \in I \times I$ there is positive integer $m$ such that $a_{i j}^{(m)}>0$, where $a_{i j}^{(m)}$ is the entry of the matrix $A^{m}$.

Given $I$ and the irreducible incidence matrix $A$ as above, we denote

$$
\Sigma_{A}=\left\{\left(x_{k}\right)_{k \geq 0}: x_{k} \in I, A_{x_{k}, x_{k+1}}=1, k \geq 0\right\}
$$

which is the corresponding subshift space, and let $\sigma$ be the shift transformation on $\Sigma_{A}$. We equip $\Sigma_{A}$ with a metric $d_{f}$ depending on the dynamics which is defined as follows. First for $i, j \in I, i \neq j$ let $\kappa(i, j)$ be the integer such that $\left|a_{i}-a_{j}\right|_{p}=p^{-\kappa(i, j)}$. It clear that $\kappa(i, j)<-\log _{p}(r)$, where $r$ is the radius of the balls at the beginning of section 2.3. By the ultra-metric inequality, we have

$$
|x-y|_{p}=\left|a_{i}-a_{j}\right|_{p} \quad i \neq j, \forall x \in B_{r}\left(a_{i}\right), \forall y \in B_{r}\left(a_{j}\right)
$$

For $x=\left(x_{0}, x_{1}, \ldots, x_{n}, \ldots\right) \in \Sigma_{A}$ and $y=\left(y_{0}, y_{1}, \ldots, y_{n}, \ldots\right) \in \Sigma_{A}$, define

$$
d_{f}(x, y)= \begin{cases}p^{-\tau_{x_{0}}-\tau_{x_{1}}-\cdots-\tau_{x_{n-1}}-\kappa\left(x_{n}, y_{n}\right)} & , \text { if } n \neq 0 \\ p^{-\kappa\left(x_{0}, y_{0}\right)} & , \text { if } n=0\end{cases}
$$

where $n=n(x, y)=\min \left\{i \geq 0: x_{i} \neq y_{i}\right\}$. It is clear that $d_{f}$ defines the same topology as the classical metric which is defined by $d(x, y)=p^{-n(x, y)}$.

Theorem 2.4. ([12]) Let $\left(X, J_{f}, f\right)$ be a transitive $p$-adic weak repeller with incidence matrix $A$. Then the dynamics $\left(J_{f}, f,|\cdot|_{p}\right)$ is isometrically conjugate to the shift dynamics $\left(\Sigma_{A}, \sigma, d_{f}\right)$. 


\section{Description AND EXISTENCE OF THE FIXED POINTS}

In this section we consider a dynamical system corresponding to a non-linear function $f_{a, b}: \mathbb{Q}_{p} \rightarrow \mathbb{Q}_{p}$ given by

$$
f_{a, b}(u)=\left(\frac{(a b u)^{2}+1}{b^{2}+a^{2} u^{2}}\right), \quad a, b \in \mathcal{E}_{p} .
$$

We stress that the mentioned function has recently been studied in [32] to find $p$-adic Gibbs measures. To investigate other properties of the Gibbs measures it is needed to study dynamics of $f_{a, b}$.

First, recall that two functions $f$ and $g$ are topologically conjugate if there exists a homeomorphism $h$ such that $h \circ f=g \circ h$. Moreover, if $h$ is isometry, then the functions $f$ and $g$ are called isometrically conjugate. It is clear that the topological conjugacy is weak that isometrical conjugacy, since the function $h$ is not necessary to be an isometry in general.

One can prove the following fact.

Proposition 3.1. The function $f_{a, b}$ is topologically conjugate to

$$
g_{a, b}(u)=a\left(\frac{b^{2} u^{2}+1}{b^{2}+u^{2}}\right) .
$$

Note that the conjugation in the last proposition is isometric conjugacy.

The function (3.2) is called a generalized Ising mapping. In what follows, as we mentioned, we always assume $p \geq 3$ without stressing it.

We will investigate the function $g_{a, b}$. Some proofs of the relations between the coefficients $a, b$ and the fixed points of $g_{a, b}$ are given in [32]. For the sake of completeness, we are going to prove auxiliary facts.

Proposition 3.2. Let $p \geq 3$ and $a, b \in \mathcal{E}_{p}$. Then the following statements are true:

(i) $g_{a, b}\left(\mathcal{E}_{p}\right) \subset \mathcal{E}_{p}$,

(ii) $g_{a, b}$ is a contraction on $\mathcal{E}_{p}$,

Proof. (i) To prove this let us examine $\left|g_{a, b}(u)-1\right|_{p} \leq \frac{1}{p}$, for all $u \in \mathcal{E}_{p}$. Indeed, from $a, b \in \mathcal{E}_{p}$, $\left|a b^{2}-1\right|_{p}<1,\left|b^{2}-1\right|_{p}<1$, and $|2|_{p}=1$, one gets

$$
\begin{aligned}
\left|g_{a, b}(u)-1\right|_{p} & =\frac{\left|a b^{2} u^{2}+a-b^{2}-u^{2}\right|_{p}}{\left|b^{2}+u^{2}\right|_{p}} \\
& =\left|\left(a b^{2}-1\right) u^{2}+a-1-\left(b^{2}-1\right)\right|_{p} \\
& \leq \frac{1}{p} .
\end{aligned}
$$

(ii) Now we show that the function $g_{a, b}$ is a contraction on $\mathcal{E}_{p}$. Indeed,

$$
\begin{aligned}
\left|g_{a, b}(x)-g_{a, b}(y)\right|_{p} & =\frac{\left|\left(b^{4}-1\right)\left(x^{2}-y^{2}\right)\right|_{p}}{\left|\left(b^{2}+x^{2}\right)\left(b^{2}+y^{2}\right)\right|_{p}} \\
& =\left|b^{4}-1\right|{ }_{p}\left|x^{2}-y^{2}\right|_{p} \\
& \leq \frac{1}{p}|x-y|_{p}
\end{aligned}
$$

From this proposition due to the Banach Contraction Principle we infer that there exists $x_{0} \in \mathcal{E}_{p}$ such that $g_{a, b}\left(x_{0}\right)=x_{0}$.

Now we are going to describe all fixed points of $g_{a, b}$. 
Theorem 3.3. Let $a, b \in \mathcal{E}_{p}$ with $b \neq 1$, and $g_{a, b}$ be given by (3.2). Then the following statements hold:

(i) the function $g_{a, b}$ has a unique fixed point $x_{0}$ in $\mathcal{E}_{p}$;

(ii) if $p \equiv 3(\bmod 4)$, then $x_{0}$ is the only fixed point of $g_{a, b}$. If $p \equiv 1(\bmod 4)$, then $g_{a, b}$ has at two fixed points $x_{1}, x_{2}$ different from $x_{0}$;

(iii) let $x_{1}, x_{2}$ be two fixed points of $g_{a, b}$ different from $x_{0}$. Then $x_{1}, x_{2} \in \mathbb{Q}_{p} \backslash \mathcal{E}_{p}$.

Proof. (i) By Proposition 3.2 we conclude that $g_{a, b}$ satisfies the Banach Contraction principle on $\mathcal{E}_{p}$. Therefore, $g_{a, b}$ has a unique fixed point belonging to $\mathcal{E}_{p}$, i.e. there exists $x_{0} \in \mathcal{E}_{p}$ such that $g_{a, b}\left(x_{0}\right)=x_{0}$.

(ii) Consider the equation $x=g_{a, b}(x)$, which can be rewritten as follows

$$
x^{3}-a b^{2} x^{2}+b^{2} x-a=0
$$

Note that, in general, we may solve the last equation by methods developed in [33], but those methods give only information about the existence of solutions. In reality, we need more properties of the solutions (see further sections). Therefore, we are going to find all the solutions.

Due to (i) we know that one of solutions of the last equation is $x_{0}$. Therefore, one can write

$$
x^{3}-a b^{2} x^{2}+b^{2} x-a=\left(x-x_{0}\right)\left(x^{2}+\left(x_{0}-a b^{2}\right) x+\left(x_{0}^{2}-a b^{2} x_{0}+b^{2}\right)\right) .
$$

The equality (3.3) yields that

$$
\frac{a}{x_{0}}=x_{0}^{2}-a b^{2} x_{0}+b^{2} .
$$

Hence, the quadratic equation of RHS of (3.3) can be rewritten as follows

$$
x^{2}+\left(x_{0}-a b^{2}\right) x+\frac{a}{x_{0}}=0 .
$$

For us it is enough to solve (3.4). Let us find its discriminant

$$
\begin{aligned}
\triangle & =x_{0}^{2}-2 a b^{2} x_{0}+a^{2} b^{4}-4 \frac{a}{x_{0}} \\
& =x_{0}^{2}-2 a b^{2} x_{0}+a^{2} b^{4}-4\left(x_{0}^{2}-a b^{2} x_{0}+b^{2}\right) \\
& =-3 x_{0}^{2}+2 a b^{2} x_{0}-4 b^{2}+a^{2} b^{4},
\end{aligned}
$$

here we have used the equality $\frac{a}{x_{0}}=x_{0}^{2}-a b^{2} x_{0}+b^{2}$.

By using simple calculations, we find

$$
\begin{aligned}
\triangle & =-4-4\left(x_{0}-1\right)+4(a-1)\left(b^{2}-1\right)+4(a-1) \\
& +2\left(x_{0}-1\right)\left(a b^{2}-1\right)-3\left(x_{0}-1\right)^{2}+\left(a b^{2}-1\right)^{2} .
\end{aligned}
$$

Now, taking into account $a, b, x_{0} \in \mathcal{E}_{p}$, one gets

$$
\triangle=-4+p^{n} \delta
$$

According to Lemma 2.1 we conclude that $\sqrt{\triangle}$ exists if and only if $\sqrt{-4}$ exists, which is equivalent the existence of $\sqrt{-1}$. Taking into account the fact that $\sqrt{-1}$ exists in $\mathbb{Q}_{p}$ if and only if $p \equiv 1(\bmod 4)$.

(iii) Assume that (3.4) has two solutions $x_{1}, x_{2}$. So Viete's theorem implies that

$$
\left\{\begin{array}{l}
x_{1}+x_{2}=a b^{2}-x_{0} \\
x_{1} \cdot x_{2}=\frac{a}{x_{0}}
\end{array}\right.
$$

From $\left|x_{0}-a b^{2}\right|_{p} \leq \frac{1}{p}$ we get $\left|x_{1}\right|_{p}\left|x_{2}\right|_{p}=1,\left|x_{1}+x_{2}\right|_{p} \leq \frac{1}{p}$. Hence, we obtain $\left|x_{1}\right|_{p}=1,\left|x_{2}\right|_{p}=1$.

Note that the solutions have the following form

$$
x_{1,2}=\frac{a b^{2}-x_{0} \pm \sqrt{\triangle}}{2} .
$$


From (3.5) one can see that

$$
\begin{aligned}
|(\sqrt{\triangle}-2)(\sqrt{\triangle}+2)|_{p} & =|\triangle-4|_{p} \\
& =\left|-8+p^{-\gamma_{3}} \varepsilon_{3}\right|_{p} \\
& =1 .
\end{aligned}
$$

Hence, one gets that

$$
|\sqrt{\triangle}-2|_{p}=1,|\sqrt{\triangle}+2|_{p}=1 .
$$

Therefore, from (3.9) together with $\left|a b^{2}-1\right|_{p}<1,\left|x_{0}-1\right|_{p}<1$ we have

$$
\begin{aligned}
\left|x_{1,2}-1\right|_{p} & =\left|a b^{2}-x_{0} \pm \sqrt{\triangle}-2\right|_{p} \\
& =\left|a b^{2}-1-\left(x_{0}-1\right) \pm \sqrt{\triangle}-2\right|_{p} \\
& =1
\end{aligned}
$$

which means $x_{1,2} \notin \mathcal{E}_{p}$. This completes the proof.

Lemma 3.4. Let $a, b \in \mathcal{E}_{p}$ with $b \neq 1$, and $g_{a, b}$ be given by (3.2). Assume that $x_{0}, x_{1}, x_{2}$ are fixed points of $g_{a, b}$. Then the following statements hold:

(i) $\left|x_{0}-a\right|_{p}<|b-1|_{p}$;

(ii) $\left|b^{2}-1+\left(b^{2} a-x_{0}\right) x_{1,2}\right|_{p}=|b-1|_{p}$;

(iii) $\left|x_{1,2}^{2}+b^{2}\right|_{p}=|b-1|_{p}$;

(iv) $\left|x_{0}^{2}+b^{2}\right|_{p}\left|x_{0}^{2} b^{2}+1\right|_{p}=1$;

(v) $\left|x_{1,2}^{2}+b^{2}\right|_{p}\left|x_{1,2}^{2} b^{2}+1\right|_{p}=\left|x_{1,2}^{2}+b^{2}\right|_{p}^{2} \leq \frac{1}{p^{2}}$.

(vi) $\left|x_{0}-1\right|_{p}<|b-1|_{p}$, if $|a-1|_{p}<|b-1|_{p}$;

(vii) if $|a-1|_{p}<|b-1|_{p}$, then

$$
\triangle=-4+p^{2 m+l} \delta
$$

where $b-1=p^{m} \varepsilon, l \in \mathbb{N}$ and $\delta \in \mathbb{Z}_{p}$.

Proof. (i) From Lemma 2.3 we find

$$
\begin{aligned}
\left|x_{0}-a\right|_{p} & =\left|g\left(x_{0}\right)-a\right|_{p} \\
& =\frac{\left|b^{2} x_{0}^{2}+1-b^{2}-x_{0}^{2}\right|_{p}}{\left|b^{2}+x_{0}^{2}\right|_{p}} \\
& =\left|x_{0}^{2}-1\right|_{p}|b-1|_{p} \\
& =\left|x_{0}-1\right|_{p}|b-1|_{p}
\end{aligned}
$$

Due to $\left|x_{0}-1\right|_{p}<1$ the last expression implies the desired inequality.

(ii) We first observe that from (3.9) similarly to (3.10) one can get $\left|x_{1,2}+1\right|_{p}=1$. This together with the strong triangle inequality yields that

$$
\left|a x_{1,2}+1\right|_{p}=\left|1+x_{1,2}+(a-1) x_{1,2}\right|_{p}=1
$$

here we have used that $|a-1|_{p}<1$.

Now taking into account (i) and (3.11) we obtain

$$
\begin{aligned}
\left|b^{2}-1+\left(b^{2} a-x_{0}\right) x_{1,2}\right|_{p} & =\left|b^{2}-1+\left(b^{2}-1\right) a x_{1,2}-\left(x_{0}-a\right) x_{1,2}\right|_{p} \\
& =\left|\left(b^{2}-1\right)\left(1+a x_{1,2}-\left(x_{0}-a\right) x_{1,2}\right)\right|_{p} \\
& =\left|b^{2}-1\right|_{p} \\
& =|b-1|_{p} .
\end{aligned}
$$


(iii) Due to $x_{1,2}^{2}=\left(a b^{2}-x_{0}\right) x_{1,2}-\frac{a}{x_{0}}$ and taking into account (i) and (ii) one finds

$$
\begin{aligned}
\left|b^{2}+x_{1,2}^{2}\right|_{p} & =\left|b^{2}+\left(a b^{2}-x_{0}\right) x_{1,2}-\frac{a}{x_{0}}\right|_{p} \\
& =\left|b^{2}-1+\left(a b^{2}-x_{0}\right) x_{1,2}-\frac{1}{x_{0}}\left(a-x_{0}\right)\right|_{p} \\
& =|b-1|_{p} .
\end{aligned}
$$

(iv) The proof of (iv) immediately follows from Lemma 2.3.

(v) Now the fact that $x_{1,2}$ is a fixed point of $g$ together with (iii) we obtain

$$
\begin{aligned}
\left|x_{1,2}^{2}+b^{2}\right|_{p}\left|x_{1,2}^{2} b^{2}+1\right|_{p} & =\left|x_{1,2}^{2}+b^{2}\right|_{p}^{2} \frac{\left|x_{1,2}^{2} b^{2}+1\right|_{p}}{\left|x_{1,2}^{2}+b^{2}\right|_{p}} \\
& =\left|x_{1,2}^{2}+b^{2}\right|_{p}^{2}\left|x_{1,2}\right|_{p} \\
& =\left|x_{1,2}^{2}+b^{2}\right|_{p}^{2} \\
& \leq \frac{1}{p^{2}} .
\end{aligned}
$$

(vi)

$$
\begin{aligned}
\left|x_{0}-1\right|_{p} & =\left|g_{a, b}\left(x_{0}\right)-1\right|_{p} \\
& =\left|\frac{a\left(b^{2} x_{0}^{2}+1\right)}{b^{2}+x_{0}^{2}}-1\right|_{p} \\
& =\left|a\left(b^{2} x_{0}^{2}+1\right)-\left(b^{2}+x_{0}^{2}\right)\right|_{p} \\
& =\left|a\left(b^{2}-1\right)\left(x_{0}^{2}-1\right)+(a-1)\left(x_{0}^{2}-1\right)+a\left(b^{2}-1\right)+2(a-1)+\left(1-b^{2}\right)\right|_{p} \\
& = \begin{cases}|a-1|_{p}, & \text { if }|a-1|_{p} \geq|b-1|_{p} ; \\
|b-1|_{p} p^{-l}, & \text { if }|a-1|_{p}<|b-1|_{p}, l \geq 1 .\end{cases}
\end{aligned}
$$

(vii) Using the proof Lemma 3.4 (i) and $|a-1|_{p}<|b-1|_{p}$ we find $\left|a b^{2}-1\right|_{p}=|b-1|_{p}$. This together with (3.5) and Lemma 3.4 (vi) implies the required assertion.

This completes the proof.

\section{The Classification of the fiXed points of p-ADiC Dynamical System}

In this section, we study behavior of the fixed points of function (3.2). We will describe the behavior of the function (3.2) with respect to the parameters $a, b \in \mathbb{Q}_{p}$ whether the fixed points are attracting, neutral or repelling.

Theorem 4.1. Let $a, b \in \mathcal{E}_{p}$ and $g_{a, b}$ has three fixed points $x_{0}, x_{1}, x_{2}$. Then the following statements hold:

(i) $x_{0}$ is an attracting fixed point;

(ii) $x_{1}$ and $x_{2}$ are repelling fixed points.

Proof. From

$$
\left|\frac{d g_{a, b}}{d x}\left(x_{0}\right)\right|_{p}=\frac{|2 a|_{p}\left|x_{0}\right| p\left|b^{4}-1\right|_{p}}{\left|b^{2}+x_{0}^{2}\right|_{p}^{2}}=\left|b^{4}-1\right|_{p} \leq \frac{1}{p}
$$

We conclude that $x_{0}$ is attracting. 
One can calculate that

$$
\left|\frac{d g_{a, b}}{d x}\left(x_{1,2}\right)\right|_{p}=\frac{|2 a|_{p}\left|x_{1,2}\right| p\left|b^{4}-1\right|_{p}}{\left|b^{2}+x_{1,2}^{2}\right|_{p}^{2}}=\frac{\left|b^{4}-1\right|_{p}}{\left|b^{2}+x_{1,2}^{2}\right|_{p}^{2}}=\frac{|b-1|_{p}}{\left|b^{2}+x_{1,2}^{2}\right|_{p}^{2}},
$$

here we have used $\left|b^{4}-1\right|_{p}=\left|b^{2}+1\right|_{p}|b-1|_{p}|b+1|_{p}=|b-1|_{p}$. From Lemma 3.4 (iii), we find

$$
\left|\frac{d g_{a, b}}{d x}\left(x_{1,2}\right)\right|_{p}=\frac{|b-1|_{p}}{|b-1|_{p}^{2}}=\frac{1}{|b-1|_{p}} \geq p
$$

As a conclusion one gets that $x_{1}$ and $x_{2}$ are repelling fixed points.

Now we are going to describe the basin of attraction

$$
A\left(x_{0}\right)=\left\{x \in \mathbb{Q}_{p}: g_{a, b}^{n}(x) \rightarrow x_{0}\right\}
$$

of the fixed point $x_{0}$.

Now we define

$$
\begin{aligned}
& K=\left\{x \in S_{1}\left(x_{0}\right):\left|x^{2}+1\right|_{p} \leq\left|b^{2}-1\right|_{p}\right\}, \\
& R=\bigcap_{n=0} g_{a, b}^{-1}(K) .
\end{aligned}
$$

Now, we are going to describe the size of the attractor of the dynamic system.

Theorem 4.2. Let $a, b \in \mathcal{E}_{p}$. Then one has

$$
A\left(x_{0}\right)=\mathbb{Q}_{p} \backslash R .
$$

Note that $R$ is nonempty if and only if $p \equiv 1(\bmod 4)$.

Proof. Let us break the proof into three steps.

(I). According to Proposition 3.2 for any $x \in \mathcal{E}_{p}$ we infer that $x \in A\left(x_{0}\right)$ which means $\mathcal{E}_{p} \subset A\left(x_{0}\right)$. We notice that $\mathcal{E}_{p}=B_{1}\left(x_{0}\right)$.

(II) In this step we establish that if $x \notin \mathbb{Q}_{p} \backslash R$, then $x \in \mathcal{E}_{p}$. To do this, let us first assume $x \notin K$, then show $x \in \mathcal{E}_{p}$. Indeed, from the assumption we infer that $\left|x^{2}+1\right|_{p}>\left|b^{2}-1\right|_{p}$. Therefore, one gets

$$
\begin{aligned}
\left|g_{a, b}(x)-1\right|_{p} & =\frac{\left|\left(a b^{2}-1\right) x^{2}+a-b^{2}\right|_{p}}{\left|x^{2}+b^{2}\right|_{p}} \\
& =\frac{\left|\left(a b^{2}-1\right)\left(x^{2}+1\right)-(a+1)\left(b^{2}-1\right)\right|_{p}}{\left|x^{2}+1+b^{2}-1\right|_{p}} \\
& \leq \frac{\max \left\{\left|a b^{2}-1\right|_{p}\left|x^{2}+1\right|_{p},\left|b^{2}-1\right|_{p}\right\}}{\left|x^{2}+1\right|_{p}} \\
& <\frac{\left|x^{2}+1\right|_{p}}{\left|x^{2}+1\right|_{p}}=1 .
\end{aligned}
$$

Therefore, $g_{a, b}(x) \in \mathcal{E}_{p}$ which, due to case (I), implies $x \in A\left(x_{0}\right)$.

Now let $x \notin \mathbb{Q}_{p} \backslash R$. Then $g_{a, b}^{n}(x) \notin K$ for some $n \geq 0$. Hence, $g_{a, b}^{n+1}(x) \in \mathcal{E}_{p} \subset A\left(x_{0}\right)$.

(III) In this step, we show that if $x \in R$, then $x \notin A\left(x_{0}\right)$. Indeed, from $x \in R$, one finds that $g_{a, b}^{n}(x) \in K$ for all $n \geq 0$. This means that

$$
\left|\left(g_{a, b}^{n}(x)\right)^{2}+1\right|_{p} \leq\left|b^{2}-1\right|_{p}<1, \quad \forall n \in \mathbb{N} .
$$

By Lemma $2.3(3)$, we have $g_{a, b}^{n}(x) \notin \mathcal{E}_{p}$ for all $n \geq 0$. Since $\mathcal{E}_{p}$ is an open neighborhood of $x_{0}$, the iterates $g_{a, b}^{n}(x)$ do not converge to $x_{0}$ as $n \rightarrow \infty$. Hence, $x \notin A\left(x_{0}\right)$.

This completes the proof. 


\section{Chaoticity of the $p$-ADiC Dynamical Systems}

In this section, we prove that the renormalized dynamical system corresponding to the model is topologically conjugate to the symbolic shift. We show that the function $g_{a, b}$ in (3.2) is $p$-adic transitive weak repeller. Therefore, we prove that $p$-adic dynamical system associated to $g_{a, b}$ is topologically conjugate to a subshift of finite type.

In what follows, we always assume that the dynamical system $g_{a, b}$ has three fixed points $\left\{x_{0}, x_{1}, x_{2}\right\}$ (see Theorem 3.3). This, due to Theorem 3.3, means that $p \equiv 1(\bmod 4)$. For the sake of simplicity of calculation, we suppose that $|a-1|_{p}<|b-1|_{p}$ is satisfied.

Lemma 5.1. Let $r=|b-1|_{p}$, then one has $B_{r}\left(x_{1}\right) \cap B_{r}\left(x_{2}\right)=\emptyset$.

Proof. It is enough to show that $x_{2} \notin B_{r}\left(x_{1}\right)$. We know that (see (3.7))

$$
x_{1}=\frac{a b^{2}-x_{0}+\sqrt{\triangle}}{2}, \quad x_{2}=\frac{a b^{2}-x_{0}-\sqrt{\triangle}}{2} .
$$

Then one gets

$$
\left|x_{1}-x_{2}\right|_{p}=\left|\frac{a b^{2}-x_{0}+\sqrt{\triangle}}{2}-\frac{a b^{2}-x_{0}-\sqrt{\triangle}}{2}\right|_{p}=|\sqrt{\triangle}|_{p} .
$$

Due to $\triangle=-4+p^{m+l} \varepsilon$ and $|\triangle|_{p}=1$, so $|\sqrt{\triangle}|_{p}=1$. Therefore we have $\left|x_{1}-x_{2}\right|_{p}=1>|b-1|_{p}=r$, which means that $x_{2} \notin B_{r}\left(x_{1}\right)$.

This lemma allows us to take the square root of $g_{a, b}$ by the unique way on the balls $B_{r}\left(x_{1}\right), B_{r}\left(x_{2}\right)$, respectively. Therefore, one can prove the following result.

Proposition 5.2. The function $g_{a, b}$ given by (3.2) is topologically conjugate to

$$
k_{a, b}(x)=\left(\frac{a\left(b^{2} x+1\right)}{b^{2}+x}\right)^{2} .
$$

on the ball $B_{r}\left(x_{1}^{2}\right)$ (respectively, $B_{r}\left(x_{2}^{2}\right)$ ). Here as before $r=|b-1|_{p}$.

Proof. Let $s_{1}(x)=-\sqrt{x}, s_{2}(x)=\sqrt{x}$. Then using Lemma 3.4 one can prove that $s_{1}\left(\begin{array}{ll}\text { resp. } & \left.s_{2}\right)\end{array}\right.$ homeomorphically maps $B_{r}\left(x_{1}^{2}\right)$ (resp. $B_{r}\left(x_{2}^{2}\right)$ ) onto $B_{r}\left(x_{1}\right)$ (resp. $B_{r}\left(x_{2}\right)$ ). Moreover, we have $g_{a, b} \circ s_{i}=s_{i} \circ k_{a, b}$ on $B_{r}\left(x_{i}^{2}\right), i=1,2$.

Remark 5.1. Again by means of Lemma 3.4 we can establish that $K=\bar{B}_{r}\left(x_{1}\right) \cup \bar{B}_{r}\left(x_{2}\right)$, where $\bar{B}_{r}\left(x_{i}\right)=\left\{x \in \mathbb{Q}_{p}:\left|x-x_{i}\right|_{p} \leq r\right\}, i=1,2$. Let $K^{\prime}=\left\{x \in \mathbb{Q}_{p}:\left|x^{2}+1\right|_{p}<1\right\}$. Under condition the $p \equiv 1(\bmod 4)$, there exist $\alpha_{1}, \alpha_{2} \in \mathbb{Q}_{p}$ such that $\alpha_{i}^{2}=1$ for $i=1,2$. Then, one has

$$
K=\bar{B}_{r}\left(\alpha_{1}\right) \cup \bar{B}_{r}\left(\alpha_{2}\right), \quad K^{\prime}=B_{r}\left(\alpha_{1}\right) \cup B_{r}\left(\alpha_{2}\right) .
$$

Moreover, for $x, y \in B_{r}\left(\alpha_{i}\right), i=1,2$, we have

$$
\left|g_{a, b}(x)-g_{a, b}(y)\right|_{p}=\frac{|x-y|_{p}}{r} .
$$

Hence, $g_{a, b}$ is a $p$-adic weak repeller on $K^{\prime}$, but it does not appear to be locally Lipschitz on $K \backslash K^{\prime}$. Moreover, $g_{a, b}$ on $K^{\prime}$ does not yield an irreducible incidence matrix, as described in section 2.3. Therefore, we need to work with $k_{a, b}$ instead of $g_{a, b}$ to avoid these issues. Moreover, $k_{a, b}$ is not isometrically conjugate to $g_{a, b}$ on $K$. Indeed, one can see that $s(x)=x^{2}$ is invertible on $K$. Moreover, $s$ is an isometry when restricted to either $\bar{B}_{r}\left(x_{1}\right)$ or $\bar{B}_{r}\left(x_{2}\right)$. However, $s$ is not isometry on $K$ as a whole. For example, one has

$$
\left|s\left(x_{1}\right)-s\left(x_{2}\right)\right|_{p}=\left|x_{1}^{2}-x_{2}^{2}\right|_{p}=\left|a b^{2}-x_{0}\right|_{p}\left|x_{1}-x_{2}\right|_{p}<\left|x_{1}-x_{2}\right|_{p} .
$$

Hence, $g_{a, b}$ is not isometrically conjugate to $k_{a, b}$ on $K$ or $B_{r}\left(x_{1}\right) \cup B_{r}\left(x_{2}\right)$. Therefore, in what follows we require only topological conjugacy. 
Corollary 5.3. One has Fix $\left(k_{a, b}\right)=\left\{x_{0}^{2}, x_{1}^{2}, x_{2}^{2}\right\}$.

Lemma 5.4. Let $r=|b-1|_{p}$. Then

$$
\left|k_{a, b}(x)-k_{a, b}(y)\right|_{p}=\frac{|x-y|_{p}}{|b-1|_{p}^{2}}, \quad \text { for any } x, y \in B_{r}\left(x_{1}^{2}\right)
$$

and

$$
\left|k_{a, b}(x)-k_{a, b}(y)\right|_{p}=\frac{|x-y|_{p}}{|b-1|_{p}^{2}}, \text { for any } x, y \in B_{r}\left(x_{2}^{2}\right) .
$$

Proof. Let $x, y \in B_{r}\left(x_{1}^{2}\right)$. Then one gets $x=x_{1}^{2}+\gamma_{1}$ and $y=x_{1}^{2}+\gamma_{2}$, where $\left|\gamma_{1}\right|_{p}<r$ and $\left|\gamma_{2}\right|_{p}<r$.

From $x+b^{2}=x_{1}^{2}+b^{2}+\gamma_{1}$ we have $y+b^{2}=x_{1}^{2}+b^{2}+\gamma_{2}$. Due to Lemma 3.4 (iii) one finds $\left|x_{1}^{2}+b^{2}\right|_{p}=|b-1|_{p}=r$ and $|x+y|_{p}=\left|2 x_{1}^{2}+\gamma_{1}+\gamma_{2}\right|_{p}=1$. Simple, but long calculations imply that

$$
\left|\left(b^{4}+1\right)(x+y)+2 b^{2}(1+x y)\right|_{p}=|b-1|_{p} .
$$

Therefore, we obtain

$$
\begin{aligned}
\left|k_{a, b}(x)-k_{a, b}(y)\right|_{p} & =\frac{\left|\left(b^{4}-1\right)(x-y)\left(\left(b^{4}+1\right)(x+y)+2 b^{2}(1+x y)\right)\right|_{p}}{\left|\left(b^{2}+x\right)^{2}\left(b^{2}+y\right)^{2}\right|_{p}} \\
& =\frac{\left|\left(b^{4}-1\right)(x-y)\right|_{p}|b-1|_{p}}{\left|\left(b^{2}+x\right)^{2}\left(b^{2}+y\right)^{2}\right|_{p}} \\
& =\frac{|x-y|_{p}}{|b-1|_{p}^{2}} .
\end{aligned}
$$

Similarly, for any $x, y \in B_{r}\left(x_{2}^{2}\right)$ one can show that

$$
\left|k_{a, b}(x)-k_{a, b}(y)\right|_{p}=\frac{|x-y|_{p}}{|b-1|_{p}^{2}} .
$$

This completes the proof.

Lemma 5.5. Let $X=B_{r}\left(x_{1}^{2}\right) \cup B_{r}\left(x_{2}^{2}\right)$, here as before $r=|b-1|_{p}$. Then $k_{a, b}^{-1}(X) \subset X$.

Proof. We show that $k_{a, b}$ has two inverse branches on the set $X$, which are

$$
k_{1}^{-1}(x)=\left(\frac{a-b^{2} \sqrt{x}}{\sqrt{x}-a b^{2}}\right), \quad k_{2}^{-1}(x)=-\left(\frac{a+b^{2} \sqrt{x}}{\sqrt{x}+a b^{2}}\right) .
$$

First, let us show that for any $x \in X, k_{1}^{-1}(x) \in B_{r}\left(x_{1}^{2}\right)$. Indeed, we have

$$
\left|k_{1}^{-1}(x)-x_{1}^{2}\right|_{p}=\left|\frac{a-b^{2} \sqrt{x}}{\sqrt{x}-a b^{2}}-x_{1}^{2}\right|_{p}=\frac{\left|a-b^{2} \sqrt{x}-x_{1}^{2}\left(\sqrt{x}-a b^{2}\right)\right|_{p}}{\left|\sqrt{x}-a b^{2}\right|_{p}} .
$$

Now, let us compute the numerator and denominator of eq. (5.2).

$$
\begin{aligned}
&\left|\sqrt{x}-a b^{2}\right|_{p}=\left|x_{1}+p^{m / 2} \gamma-a b^{2}\right|_{p}=\left|\left(x_{1}-1\right)+p^{m / 2} \gamma+\left(1-a b^{2}\right)\right|_{p}=1, \\
&\left|a-b^{2} \sqrt{x}-x_{1}^{2}\left(\sqrt{x}-a b^{2}\right)\right|_{p}=\left|(a-1)-\left(b^{2}-1\right) \sqrt{x}+\left(1-a b^{2}\right)-\left(x_{1}^{2}+1\right)\left(\sqrt{x}-a b^{2}\right)\right|_{p} \\
&<|b-1|_{p} .
\end{aligned}
$$

Therefore, one gets $\left|k_{1}^{-1}(x)-x_{1}^{2}\right|_{p}<|b-1|_{p}$ which implies that $k_{1}^{-1}(x) \in B_{r}\left(x_{1}^{2}\right)$.

Similarly, one can show that $k_{2}^{-1}(x) \in B_{r}\left(x_{2}^{2}\right)$. Consequently, we conclude that $k_{a, b}^{-1}(X) \subset X$.

Lemma 5.6. One has $B_{r}\left(x_{i}^{2}\right) \subset k_{a, b}\left(B_{r}\left(x_{j}^{2}\right)\right), i, j \in\{1,2\}$.

Now, we can prove main result of this section. 
Theorem 5.7. Let $r=|b-1|_{p}, X=B_{r}\left(x_{1}^{2}\right) \cup B_{r}\left(x_{2}^{2}\right)$ and $k_{a, b}: X \rightarrow \mathbb{Q}_{p}$ be a function given by (5.1). Then the dynamics $\left(J_{k_{a, b}}, k_{a, b},|\cdot|_{p}\right)$ is isometrically conjugate to the shift dynamics $\left(\Sigma, \sigma, d_{k_{a, b}}\right)$.

Proof. It is enough to check that all conditions of Theorem 2.4 are satisfied. According to Lemma 5.5, one gets $k_{a, b}^{-1}(X) \subset X$. By Lemma 5.4 , the triple $\left(J_{k_{a, b}}, k_{a, b},|\cdot|_{p}\right)$ is a $p$-adic repeller. Finally, Lemma 5.6, an incidence matrix $A$ has the following form

$$
A=\left(\begin{array}{ll}
1 & 1 \\
1 & 1
\end{array}\right) .
$$

Therefore, the triple $\left(X, k_{a, b},|\cdot|_{p}\right)$ is a transitive. So, Theorem 2.4 implies that $p$-adic nonlinear rational dynamical system $\left(J_{k_{a, b}}, k_{a, b},|\cdot|_{p}\right)$ is isometrically conjugate to full shift dynamical system $\left(\Sigma_{A}, \sigma, d_{k}\right)$. This completes the proof.

It is well-known that the shift map is chaotic, and hence, from Theorem 5.7, we can infer that the function (3.2) is chaotic as well.

Remark 5.2. A main aim of the present paper is to establish of the chaos for the generalized $p$-adic Ising mapping in $\mathbb{Q}_{p}$, which is stated in Theorem 5.7. On the other hand, we notice that the results in $[12]$ can be extended for finite extensions of $\mathbb{Q}_{p}$. Therefore, further development of the results in this paper can be generalized as well for finite extensions, which will be a topic our next works.

\section{An application: Gibbs measures for the p-Adic Ising-Vannimenus model}

In this section, we study the existence of periodic $p$-adic Gibbs measures of $p$-adic Ising-Vannimenus model given in [32]. In [34] it has been studied 2-periodic p-adic Gibbs measures for the IsingVannimenus model on the Cayley tree of order two. In this section, we show that there are many kinds of periodic $p$-adic Gibbs measures (for definitions we refer to the appendix).

Let us consider a $H_{m}$-periodic function $\mathbf{h}=\left\{\mathbf{h}_{x}\right\}_{x \in V \backslash\left\{x^{0}\right\}}$. From the $H_{m}$-periodicity we infer that there is a $m$-collection of vectors $\left\{\mathbf{h}_{0}, \ldots, \mathbf{h}_{m-1}\right\}$, such that $\mathbf{h}_{x}=\mathbf{h}_{i}$, if $d\left(x, x^{0}\right) \equiv i(\bmod m)$, $i=0, \ldots, m-1$. On the invariant line, we have $\mathbf{h}_{i}=\left(h_{i}, 1, \ldots, 1\right)(i=0, \ldots, m-1)$.

Then the equation (7.11) for the $H_{m}$-periodic functions reduces to the following system

$$
h_{i}=g_{a, b}\left(h_{i+1}\right), \quad h_{m}=g_{a, b}\left(h_{0}\right), i=1, \ldots, m-1 .
$$

It is clear that the equation (6.1) is equivalent to finding $m$-periodic points of the function $g_{a, b}$. Hence, the existence of periodic orbits of the function implies the existence of $H_{m}$-periodic $p$-adic quasi Gibbs measures.

It is well-known that the shift operator has infinitely many periodic points, therefore, Theorem 5.7 implies that the function $k_{a, b}$ also has infinitely many periodic points. Also, because $g_{a, b}$ is topologically conjugate to $k_{a, b}$, the function $g_{a, b}$ also has infinitely many periodic points. Hence, there are infinitely many $H_{m}$-periodic $p$-adic quasi Gibbs measures for the $p$-adic Ising-Vannimenus model.

\section{ACKNOWLEDGMENTS}

The authors would like to thank the referees for their useful suggestions which allowed to improve the content of the paper.

\section{Appendix}

7.1. -adic measure. Let $(X, \mathcal{B})$ be a measurable space, where $\mathcal{B}$ is an algebra of subsets $X$. A function $\mu: \mathcal{B} \rightarrow \mathbb{Q}_{p}$ is said to be a $p$-adic measure if for any $A_{1}, \ldots, A_{n} \in \mathcal{B}$ such that $A_{i} \cap A_{j}=\emptyset$ $(i \neq j)$ the equality holds

$$
\mu\left(\bigcup_{j=1}^{n} A_{j}\right)=\sum_{j=1}^{n} \mu\left(A_{j}\right)
$$


A $p$-adic measure is called a probability measure if $\mu(X)=1$. One of the important conditions is boundedness, namely a $p$-adic probability measure $\mu$ is called bounded if $\sup \left\{|\mu(A)|_{p}: A \in \mathcal{B}\right\}<\infty$. For more detailed information about $p$-adic measures we refer to [2],[18],[23].

7.2. Cayley tree. Let $\Gamma_{+}^{k}=(V, L)$ be a semi-infinite Cayley tree of order $k \geq 1$ with the root $x^{0}$ (where each vertex has exactly $k+1$ edges, except for the root $x^{0}$, which has $k$ edges). Here $V$ is the set of vertices and $L$ is the set of edges. The vertices $x$ and $y$ are called nearest neighbors and they are denoted by $l=<x, y>$ if there exists an edge connecting them. A collection of the pairs $\left\langle x, x_{1}\right\rangle, \ldots,\left\langle x_{d-1}, y\right\rangle$ is called a path from the point $x$ to the point $y$. The distance $d(x, y), x, y \in V$, on the Cayley tree, is the length of the shortest path from $x$ to $y$.

Recall a coordinate structure in $\Gamma_{+}^{k}$ : every vertex $x$ (except for $x^{0}$ ) of $\Gamma_{+}^{k}$ has coordinates $\left(i_{1}, \ldots, i_{n}\right)$, here $i_{m} \in\{1, \ldots, k\}, 1 \leq m \leq n$ and for the vertex $x^{0}$ we put (0). Namely, the symbol (0) constitutes level 0 , and the sites $\left(i_{1}, \ldots, i_{n}\right)$ form level $n$ ( i.e. $d\left(x^{0}, x\right)=n$ ) of the lattice.

For $x \in \Gamma_{+}^{k}, x=\left(i_{1}, \ldots, i_{n}\right)$ put

$$
S(x)=\{(x, i): 1 \leq i \leq k\},
$$

here $(x, i)$ is short for $\left(i_{1}, \ldots, i_{n}, i\right)$. This set is called a set of direct successors of $x$.

Let us define on $\Gamma_{+}^{k}$ a binary operation $\circ: \Gamma_{+}^{k} \times \Gamma_{+}^{k} \rightarrow \Gamma_{+}^{k}$ as follows: for any two elements $x=\left(i_{1}, \ldots, i_{n}\right)$ and $y=\left(j_{1}, \ldots, j_{m}\right)$ put

$$
x \circ y=\left(i_{1}, \ldots, i_{n}\right) \circ\left(j_{1}, \ldots, j_{m}\right)=\left(i_{1}, \ldots, i_{n}, j_{1}, \ldots, j_{m}\right)
$$

and

$$
x \circ x^{0}=x^{0} \circ x=\left(i_{1}, \ldots, i_{n}\right) \circ(0)=\left(i_{1}, \ldots, i_{n}\right) .
$$

By means of the defined operation $\Gamma_{+}^{k}$ becomes a noncommutative semigroup with a unit. Using this semigroup structure one defines translations $\tau_{g}: \Gamma_{+}^{k} \rightarrow \Gamma_{+}^{k}, g \in \Gamma_{+}^{k}$ by

$$
\tau_{g}(x)=g \circ x .
$$

It is clear that $\tau_{(0)}=i d$.

Let $H \subset \Gamma_{+}^{k}$ be a sub-semigroup of $\Gamma_{+}^{k}$ and $h: \Gamma_{+}^{k} \rightarrow Y$ be a $Y$-valued function defined on $\Gamma_{+}^{k}$. We say that $h$ is $H$-periodic if $h\left(\tau_{g}(x)\right)=h(x)$ for all $g \in H$ and $x \in \Gamma_{+}^{k}$. Any $\Gamma_{+}^{k}$-periodic function is called translation invariant. For each $m \geq 2$ we put

$$
H_{m}=\left\{x \in \Gamma_{+}^{k}: d\left(x, x^{0}\right) \equiv 0(\bmod m)\right\} .
$$

One can check that $H_{m}$ is a sub-semigroup.

Let us set

$$
W_{n}=\left\{x \in V \mid d\left(x, x^{0}\right)=n\right\}, \quad V_{n}=\bigcup_{m=1}^{n} W_{m}, \quad L_{n}=\left\{l=<x, y>\in L \mid x, y \in V_{n}\right\} .
$$

Two vertices $x, y \in V^{0}$ are called the next-nearest neighbors if $d(x, y)=2$. The next-nearestneighbors vertices $x$ and $y$ are called the prolonged next-nearest neighbors if $x \in W_{n-2}$ and $y \in W_{n}$ for some $n \geq 1$, which are denoted by $>x, y<$. The next-nearest-neighbor vertices $x$ and $y$ are called one-level next-nearest-neighbors if $x, y \in W_{n}$ for some $n$ and they are denoted by $>\overline{x, y}<$.

7.3. $p$-adic Ising-Vannimenus (IV) model and its $p$-adic Gibbs measures. In this subsection we consider the $p$-adic Ising-Vannimenus model given in [32] where spin takes values in the set $\Phi=$ $\{-1,+1\},\left(\Phi\right.$ is called a state space) and is assigned to the vertices of the tree $\Gamma_{+}^{k}=(V, L)$. A configuration $\sigma$ on $V$ is then defined as a function $x \in V \rightarrow \sigma(x) \in \Phi$; in a similar manner one defines configurations $\sigma_{n}$ and $\omega$ on $V_{n}$ and $W_{n}$, respectively. The set of all configurations on $V$ (resp. $V_{n}$, 
$\left.W_{n}\right)$ coincides with $\Omega=\Phi^{V}$ (resp. $\left.\Omega_{V_{n}}=\Phi^{V_{n}}, \quad \Omega_{W_{n}}=\Phi^{W_{n}}\right)$. One can see that $\Omega_{V_{n}}=\Omega_{V_{n-1}} \times \Omega_{W_{n}}$. Using this, for given configurations $\sigma_{n-1} \in \Omega_{V_{n-1}}$ and $\omega \in \Omega_{W_{n}}$ we define their concatenations by

$$
\left(\sigma_{n-1} \vee \omega\right)(x)= \begin{cases}\sigma_{n-1}(x), & \text { if } x \in V_{n-1}, \\ \omega(x), & \text { if } x \in W_{n}\end{cases}
$$

It is clear that $\sigma_{n-1} \vee \omega \in \Omega_{V_{n}}$.

The Hamiltonian $H_{n}: \Omega_{V_{n}} \rightarrow \mathbb{Q}_{p}$ of the $p$-adic Ising-Vannimenus model has a form

$$
H_{n}(\sigma)=J \sum_{<x, y>\in L_{n}} \sigma(x) \sigma(y)+J_{1} \sum_{>x, y<: x, y \in V_{n}} \sigma(x) \sigma(y)+J_{0} \sum_{>\overline{x, y<: x, y \in V_{n}}} \sigma(x) \sigma(y)
$$

where $J_{1}, J, J_{0} \in B\left(0, p^{-1 /(p-1)}\right)$ are coupling constants.

Note that the last condition together with the strong triangle inequality implies the existence of $\exp _{p}\left(H_{n}(\sigma)\right)$ for all $\sigma \in \Omega_{V_{n}}, n \in \mathbb{N}$. This is required to our construction.

Assume that $\mathbf{h}: V \backslash\left\{x^{(0)}\right\} \times V \backslash\left\{x^{(0)}\right\} \rightarrow \mathbb{Q}_{p}^{\Phi \times \Phi}$ be a mapping i.e. $\{x, y\} \rightarrow \mathbf{h}_{x y}$, where $\mathbf{h}_{x y}=$ $\left(h_{x y,--}, h_{x y,-+}, h_{x y,+-}, h_{x y,++}\right), h_{x y, \pm \pm} \in \mathbb{Q}_{p}$ and $x, y \in V \backslash\left\{x^{(0)}\right\}$.

Given $n \in \mathbb{N}$, let us consider a $p$-adic probability measure $\mu_{\mathbf{h}}^{(n)}$ on $\Omega_{V_{n}}$ defined by

$$
\mu_{\mathbf{h}}^{(n)}(\sigma)=\frac{1}{Z_{n}^{(\mathbf{h})}} \exp _{p}\left(H_{n}(\sigma)\right) \prod_{x \in W_{n-1}, y \in S(x)}\left(h_{x y, \sigma(x) \sigma(y)}\right)^{\sigma(x) \sigma(y)}
$$

Here, $\sigma \in \Omega_{V_{n}}$, and $Z_{n}^{(\mathbf{h})}$ is the corresponding normalizing factor called a partition function given by

$$
Z_{n}^{(\mathbf{h})}=\sum_{\sigma \in \Omega_{V_{n}}} \exp _{p}\left(H_{n}(\sigma)\right) \prod_{x \in W_{n-1}, y \in S(x)}\left(h_{x y, \sigma(x) \sigma(y)}\right)^{\sigma(x) \sigma(y)} .
$$

We recall [28] that one of the central results of the theory of probability concerns a construction of an infinite volume distribution with given finite-dimensional distributions, which is a well-known Kolmogorov's extension Theorem [40]. In this paper we apply the Kolmogorov's Theorem in a $p$-adic context [22]. Namely, a $p$-adic probability measure $\mu$ on $\Omega$, which is compatible with defined ones $\mu_{\mathbf{h}}^{(n)}$, i.e.

$$
\mu\left(\sigma \in \Omega:\left.\sigma\right|_{V_{n}}=\sigma_{n}\right)=\mu_{\mathbf{h}}^{(n)}\left(\sigma_{n}\right), \quad \text { for all } \sigma_{n} \in \Omega_{V_{n}}, n \in \mathbb{N},
$$

exists if the measures $\mu_{\mathbf{h}}^{(n)}, n \geq 1$ satisfy the compatibility condition, i.e.

$$
\sum_{\omega \in \Omega_{W_{n}}} \mu_{\mathbf{h}}^{(n)}(\sigma \vee \omega)=\mu_{\mathbf{h}}^{(n-1)}(\sigma), \text { for all } \sigma \in \Omega_{V_{n-1}} .
$$

We should stress that using the compatibility condition for the Ising model on the Bethe lattice, in the real case (see [36] for review).

Following [28] if for some function $\mathbf{h}$ the measures $\mu_{\mathbf{h}}^{(n)}$ satisfy the compatibility condition, then there is a unique $p$-adic probability measure, which we denote by $\mu_{\mathbf{h}}$, since it depends on $\mathbf{h}$. Such a measure $\mu_{\mathbf{h}}$ is said to be a p-adic quasi Gibbs measure corresponding to the p-adic Ising-Vannimenus model. By $Q \mathcal{G}(H)$ we denote the set of all $p$-adic quasi Gibbs measures associated with functions $\mathbf{h}=\left\{\mathbf{h}_{x}, x \in V\right\}$. If there are at least two distinct $p$-adic quasi Gibbs measures $\mu, \nu \in Q \mathcal{G}(H)$ such that $\mu$ is bounded and $\nu$ is unbounded, then we say that a phase transition occurs. By another words, one can find two different functions $\mathbf{s}$ and $\mathbf{h}$ defined on $\mathbb{N}$ such that there exist the corresponding measures $\mu_{\mathbf{s}}$ and $\mu_{\mathbf{h}}$, for which one is bounded, another one is unbounded. Moreover, if there is a sequence of sets $\left\{A_{n}\right\}$ such that $A_{n} \in \Omega_{V_{n}}$ with $\left|\mu\left(A_{n}\right)\right|_{p} \rightarrow 0$ and $\left|\nu\left(A_{n}\right)\right|_{p} \rightarrow \infty$ as $n \rightarrow \infty$, then we say that there occurs a strong phase transition. 
Now one can ask for what kind of functions $\mathbf{h}$ the measures $\mu_{\mathbf{h}}^{(n)}$ defined by (7.7) would satisfy the compatibility condition (7.10). The following theorem gives an answer to this question.

Theorem 7.1. [32] The measures $\mu_{\mathbf{h}}^{(n)}, n=1,2, \ldots$ (see (7.7)) satisfy the compatibility condition (7.10) if and only if for any $n \in \mathbb{N}$ the following equation holds:

$$
\left\{\begin{array}{l}
h_{x y,++} \cdot h_{x y,-+}=\prod_{z \in S(y)} \frac{(a b)^{2} h_{y z,++} h_{y z,+-+1}}{a^{2} h_{y z,++} h_{y z,+-+b^{2}}} \\
h_{x y,--} \cdot h_{x y,+-}=\prod_{z \in S(y)} \frac{(a b)^{2} h_{y z,--} h_{y z,-++1}}{a^{2} h_{y z,--} h_{y z,-++b^{2}}} \\
h_{x y,++} \cdot h_{x y,+-}=\prod_{z \in S(y)} \frac{\left((a b)^{2} h_{y z,++} h_{y z,+-+1}\right) h_{y z,-+}}{\left.\left(a^{2} h_{y z,--} h_{y z,-++}\right) b^{2}\right) h_{y z,+-}}
\end{array}\right.
$$

where $a=\exp _{p}(J), b=\exp _{p}\left(J_{1}\right)$.

\section{REFERENCES}

[1] Albeverio S., Cianci R., . Khrennikov A.Yu., p-adic valued quantization, P-Adic Numbers, Ultrametric Analysis, and Applications, 1, 91-104 (2009).

[2] Anashin V., Khrennikov A., Applied Algebraic Dynamics, Walter de Gruyter, Berlin, New York, 2009.

[3] Areféva I. Ya., Dragovic B., Frampton P.H., Volovich I.V. The wave function of the Universe and $p-$ adic gravity, Int. J. Modern Phys. A 6(1991) 4341-4358.

[4] Benedetto R., Reduction, dynamics, and Julia sets of rational functions, J. Number Theory, 86 (2001), 175195.

[5] Benedetto R., Hyperbolic maps in p-adic dynamics, Ergod. Th.\&3 Dynam.Sys. 21(2001), 1-11.

[6] Bosco F. A., Goulart R. S. Jr Fractal dimension of the Julia set associated with the Yang-Lee zeros of the Ising model on the Cayley tree, Europhys. Let. 4(1987) 1103-1108.

[7] Diao H., Silva C.E., Digraph representations of rational functions over the $p$-adic numbers, p-Adic Numbers, Ultametric Anal. Appl. 3(2011), 23-38.

[8] Dragovich B., Khrennikov A.Yu., Kozyrev S.V., Volovich I.V. On p-adic mathematical physics, p-Adic Numbers, Ultrametric Analysis and Appl. 1 (2009), 1-17.

[9] Eggarter T.P. Cayley trees, the Ising problem, and the thermodynamic limit, Phys. Rev. B 9(1974) 2989-2992.

[10] Gyorgyi G., Kondor I., Sasvari L., Tel T. From phase transitions to chaos, World Scientific, Singapore, 1992.

[11] Herman M., Yoccoz J.-C., Generalizations of some theorems of small divisors to non-Archimedean fields, In: Geometric Dynamics (Rio de Janeiro, 1981), Lec. Notes in Math. 1007, Springer, Berlin, 1983, pp.408-447.

[12] Fan A.H., Liao L.M., Wang Y.F., Zhou D., p-adic repellers in $Q_{p}$ are subshifts of finite type, C. R. Math. Acad. Sci Paris, 344 (2007), 219-224.

[13] Fan A.H., Fan S.L., Liao L.M., Wang Y.F. On minimal deecomposition of $p$-adic homographic dynamical systems, Adv. Math. 257(2014), 92-135.

[14] Fan A.H., Fan S.L., Liao L.M., Wang Y.F. Minimality of $p$-adic rational maps with good reduction, Discrete Cont. Dyn. Sys. 37(2017), 3161-3182.

[15] Fisher M.E., The renormalization group in the theory of critical behavior, Rev. Mod. Phys. 46(1974), 597-616.

[16] Kaplan S., A Survey of symbolic dynamics and celestial mechanics, Qualitative Theor. Dyn. Sys. 7 (2008), 181-193

[17] Khamraev M., Mukhamedov F.M., On a class of rational p-adic dynamical systems, J. Math. Anal. Appl. 315(2006), 76-89.

[18] Khrennikov A.Yu. p-adic valued probability measures, Indag. Mathem. N.S. 7(1996) 311-330.

[19] Khrennikov A.Yu., p-adic description of chaos. In: Nonlinear Physics: Theory and Experiment. Editors E. Alfinito, M. Boti. WSP, Singapore, 1996, pp. 177-184.

[20] Khrennikov A.Yu. p-adic Valued Distributions in Mathematical Physics, Kluwer Academic Publisher, Dordrecht, 1994.

[21] Khrennikov A., Kozyrev S.V., Oleschko, K., Jaramillo, A.G., de Jesus Correa Lopez, M., Application of p-adic analysis to time series, Inf. Dim. Anal. Quantum Probab. Related Topics 16(2013), 1350030.

[22] Khrennikov A.Yu., Ludkovsky S. Stochastic processes on non-Archimedean spaces with values in non-Archimedean fields, Markov Process. Related Fields 9(2003) 131-162.

[23] Khrennikov A.Yu., Nilsson M. p-adic deterministic and random dynamical systems, Kluwer, Dordreht, 2004.

[24] Khrennikov A., Oleschko, K. de Jess Correa Lopez M., Modeling fluids dynamics with master equations in ultrametric spaces representing the treelike structure of capillary networks. Entropy 18(2016)(7) 249. 
[25] Koblitz N. p-adic numbers, p-adic analysis and zeta-function, Berlin, Springer, 1977.

[26] Lubin J., Nonarchimedean dynamical systems, Composito Math. 94(1994), 321-346.

[27] Monroe J. L. Julia sets associated with the Potts model on the Bethe lattice and other recursively solved systems, J. Phys. A: Math. Gen. 34 (2001), 6405-6412

[28] Mukhamedov F., On dynamical systems and phase transitions for $Q+1$-state $p$-adic Potts model on the Cayley tree, Math. Phys. Anal. Geom. 53 (2013) 49-87.

[29] Mukhamedov F., Renormalization method in p-adic $\lambda$-model on the Cayley tree, Int. J. Theor. Phys. 54 (2015), 3577-3595.

[30] Mukhamedov F., Khakimov O., Phase transition and chaos: p-adic Potts model on a Cayley tree, Chaos, Solitons \& Fractals $\mathbf{8 7}(2016), 190-196$.

[31] Mukhamedov F., Khakimov O., On generalized self-similarity in p-adic field, Fractals 24(2016), No. 4, 16500419.

[32] Mukhamedov F., Dogan M., Akın H., Phase transition for the $p$-adic Ising-Vannimenus model on the Cayley tree, J. Stat. Mech. 2014) P10031.

[33] Mukhamedov F., Omirov B., Saburov M., On cubic equations over p-adic fields, Inter. J. Number Theory 10(2014), $1171-1190$.

[34] Mukhamedov F., Saburov M., Khakimov O., On p-adic Ising-Vannimenus model on an arbitrary order Cayley tree, J. Stat. Mech. (2015), P05032.

[35] Rivera-Letelier J. Dynamics of rational functions over local fields, Astérisque, 287 (2003), $147-230$.

[36] Rozikov U.A. Gibbs Measures on Cayley Trees, World Scientific, 2013.

[37] Rozikov U. A., Khakimov O. N. p-Adic Gibbs measures and Markov random fields on countable graphs Theor. Math. Phys. 175 (2013), 518-525.

[38] Rozikov U. A., Sattarov I.A., On a nonlinear p-adic dynamical systems, p-adic Numbers, Ultrametric Anal. Appl. 6 (2014), 54-65.

[39] Silverman J.H. The arithmetic of dynamical systems, Graduate Texts in Mathematics 241, New York, Springer, 2007.

[40] Shiryaev A.N. Probability, Nauka, Moscow, 1980.

[41] Thiran E., Verstegen D., Weters J. p-adic dynamics, J. Stat. Phys. 54(1989), 893-913.

[42] Vannimenus J. Modulated phase of an Ising system with competing interactions on a Cayley tree, Z. Phys. B 43 (1981) 141-145.

[43] Vladimirov V.S., Volovich I.V., Zelenov E.I. p-adic Analysis and Mathematical Physics, World Scientific, Singapour, 1994.

[44] Yurova Axelsson E., On recent results of ergodic property for p-adic dynamical systems, p-Adic Numbers, Ultametric Anal. Appl. 6(2014), 234-255.

[45] Wilson K.G., Kogut J., The renormalization group and the $\epsilon$ - expansion, Phys. Rep. 12 (1974), 75-200.

[46] Woodcock C.F., Smart N.P., p-adic chaos and random number generation, Experiment Math. 7 (1998) 333-342.

Farrukh Mukhamedov, Department of Mathematical Sciences, College of Science, The United Arab Emirates University, P.O. Box, 15551, Al Ain, Abu Dhabi, UAE

E-mail address: far75m@gmail.com farrukh.m@uaeu.ac.ae

Hasan Akin, Ceyhun Atuf Caddesi 1164. Sokak 9/ 4 Ankaya, Ankara, Turkey.

E-mail address: akinhasan25@gmail.com

Mutlay Dogan, Department of Mathematics, Ishik University, IraQ 